\title{
Editorial
}

\section{Direct Dark Matter Search}

\section{Anselmo Meregaglia, ${ }^{1}$ Davide Franco, ${ }^{2}$ Marcello Messina, ${ }^{3}$ Claudio Montanari, ${ }^{4}$ and Francesco Pietropaolo ${ }^{5}$}

\author{
${ }^{1}$ IPHC, CNRS/IN2P3, 67037 Strasbourg, France \\ ${ }^{2}$ APC, Université Paris Diderot, CNRS/IN2P3, CEA/IRFU, Observatoire de Paris, Sorbonne Paris Cité, 75205 Paris, France \\ ${ }^{3}$ Physics Department, Columbia University, Astrophysics Laboratory, 550 W 120th Street, New York, NY 10027, USA \\ ${ }^{4}$ INFN Sezione di Pavia, Via Bassi 6, 27100 Pavia, Italy \\ ${ }^{5}$ INFN Sezione di Padova, Via Marzolo 8, 35131 Padova, Italy
}

Correspondence should be addressed to Anselmo Meregaglia; anselmo.meregaglia@cern.ch

Received 3 July 2014; Accepted 3 July 2014

\begin{abstract}
Copyright (C) 2015 Anselmo Meregaglia et al. This is an open access article distributed under the Creative Commons Attribution License, which permits unrestricted use, distribution, and reproduction in any medium, provided the original work is properly cited. The publication of this article was funded by $\mathrm{SCOAP}^{3}$.
\end{abstract}

About 80 years elapsed from the first postulation by Zwicky of the existence of dark matter, as consequence of the observation of the rotational curves of cluster of elliptical galaxies. Their behavior was indeed compatible with the existence of a larger amount of gravitationally interacting matter with respect to the the visible matter. Nevertheless its nature is still nowadays an urgent open question for astrophysicist and cosmologists.

Promising candidates to solve the dark matter problem are the weakly interacting massive particles (WIMPs), which are expected to scatter off ordinary nuclei at very low rates $\left(\sim 1 /\right.$ ton/year for $\left.10^{-47} \mathrm{~cm}^{2}\right)$, yielding very low energy depositions $(<100 \mathrm{keV})$. Therefore, even if dark matter constitutes about $25 \%$ of the total energy of the universe, its direct detection is extremely challenging. In the near future, the Large Hadron Collider may have the chance to produce WIMPs in the laboratory. Furthermore, WIMPs of the galactic halo could be directly detected, in the near future, by presently running experiments, exploiting different technologies, like scintillating crystals, low temperature detectors, and noble liquids. The current status of art presents tensions among limits and results from different measurements. The next generation of experiments, thanks to the higher sensitivities and the technological improvements, will hopefully unravel the puzzle.

In this framework the authors have treated theoretical aspects, such as the halo-independent formalism and a new approach to describe the dark matter-nucleus interactions. Astronomical evidences of dark matter were addressed, as well as direct detection techniques including noble gases time projection chambers, scintillating crystals, and superheated liquid techniques.

The paper "The DarkSide multiton detector for the direct dark matter search" by C. Aalseth et al. discusses the possible use of a liquid argon time projection chamber (LAr TPC) for the detection of WIMPs in the framework of the DarkSide experiment. The project is described with particular emphasis on the next generation experiment DarkSide-G2 which plans to use a 3.6 ton LAr TPC reaching a sensitivity as low as $10^{-47} \mathrm{~cm}^{2}$ for WIMPs of $100 \mathrm{GeV} / \mathrm{c}^{2}$ mass.

The paper "Astronomical signatures of dark matter" by P. Gorenstein and W. Tucker is a review of the different astronomical observations which led to the unambiguous indirect evidence of the existence of a much larger component of nonbaryonic matter with respect to ordinary matter in the universe.

In the paper "The annual modulation signature for dark matter: DAMA/LIBRA-phase1 results and perspectives" by R. Bernabei et al., the possibility of detecting dark matter using NaI scintillating crystals is described. The results obtained with the total exposure of 1.04 ton $\times$ year collected by DAMA/LIBRA-phasel during 7 annual cycles are summarized and combined with the former DAMA/NaI data (cumulative exposure 1.33 ton $\times$ year, corresponding to 14 
annual cycles). An evidence at 9.3 $\sigma$ C.L. for the presence of dark matter particles in the galactic halo is claimed, on the basis of the exploited model independent dark matter annual modulation.

The paper "Searches for dark matter with superheated liquid techniques" by A. Pullia is a review of the direct dark matter detection based on superheated liquid techniques, including continuously sensitive bubble chambers, superheated droplet detectors (SDD), and Geysers.

In the paper "The WArP experiment: a double-phase argon detector for dark matter searches" by A. Zani the possibility of using a double-phase argon detector for the direct dark matter search is discussed in the framework of the WArP experiment. Although the experiment never performed a physics run, a technical run was taken in 2011, proving that the technology is effective in performing dark matter searches.

In the paper "New directions in direct dark matter searches" by P. Panci the status of direct dark matter detection is discussed in terms of dark matter interactions. A new approach to describe the dark matter-nucleus interactions in terms of a very limited number of relevant degrees of freedom is presented.

The paper "Halo-independent comparison of direct dark matter detection data" by E. Del Nobile is a review of the haloindependent formalism which makes it possible to compare data from different direct dark matter detection experiments without making assumptions on the properties of the dark matter halo.

Anselmo Meregaglia

Davide Franco

Marcello Messina

Claudio Montanari

Francesco Pietropaolo 

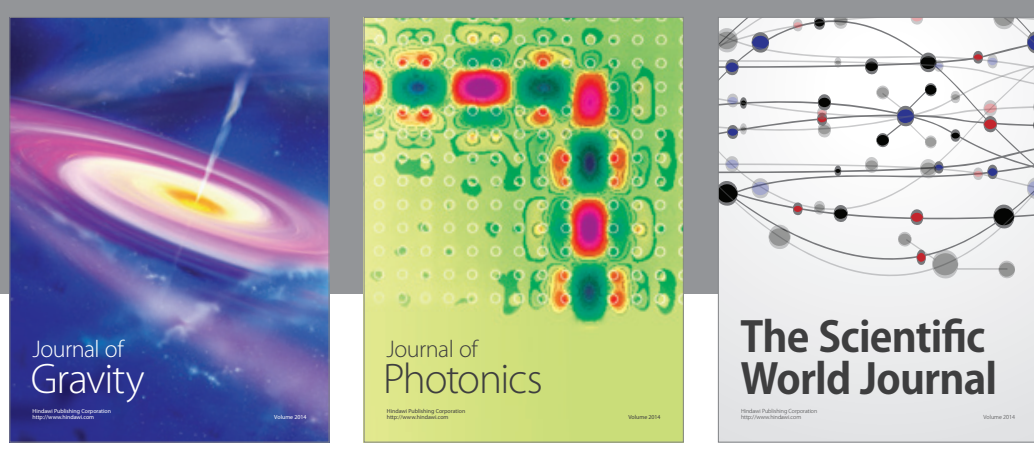

The Scientific World Journal
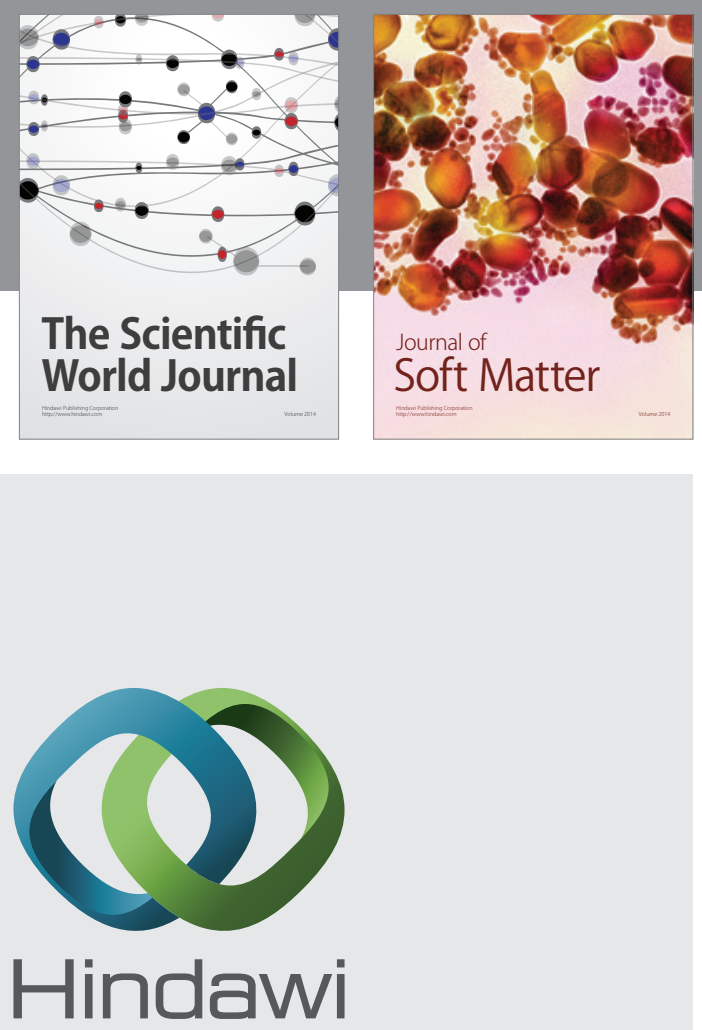

Submit your manuscripts at

http://www.hindawi.com

nternational Journal of

Statistical Mechanics
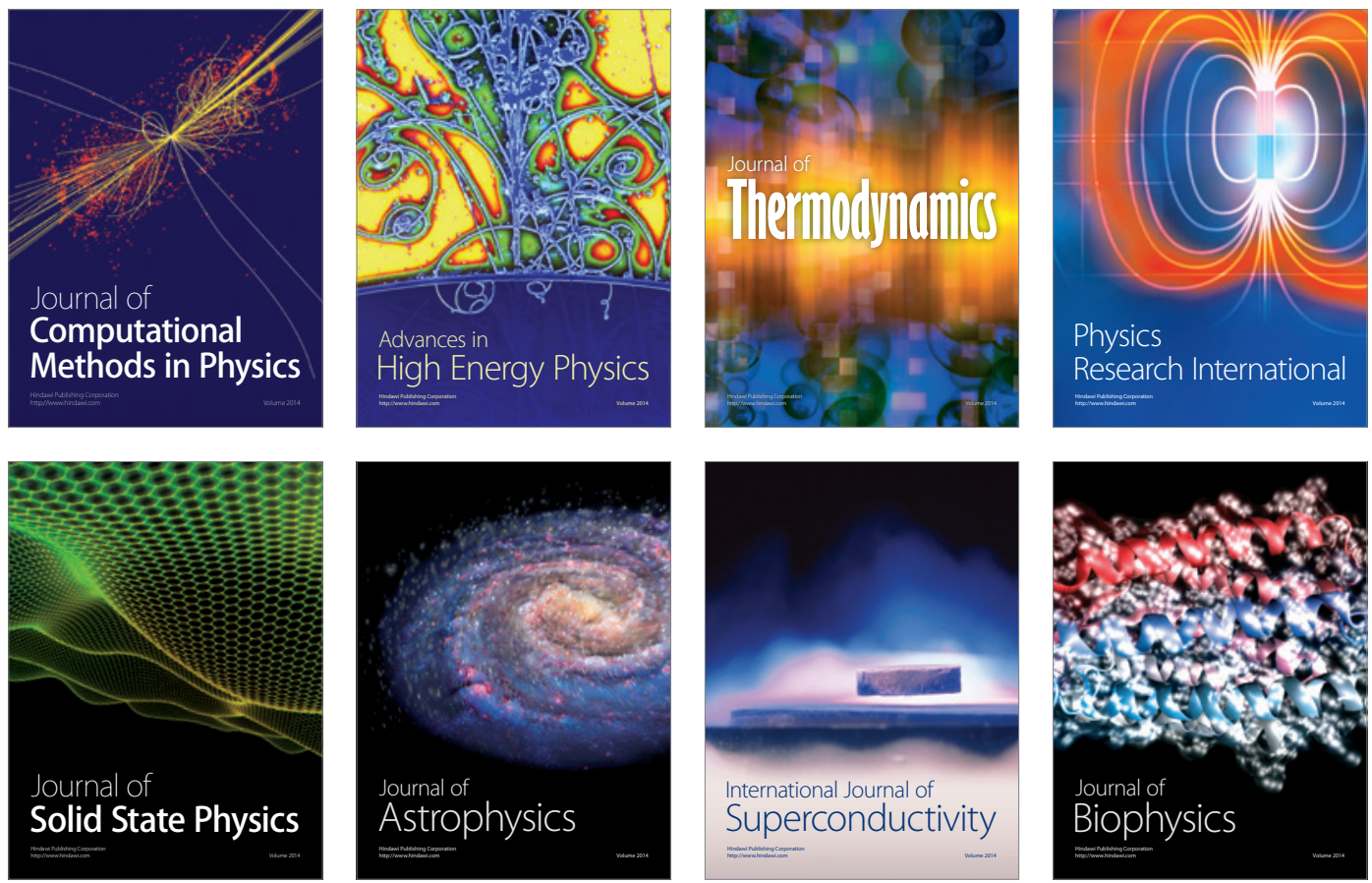
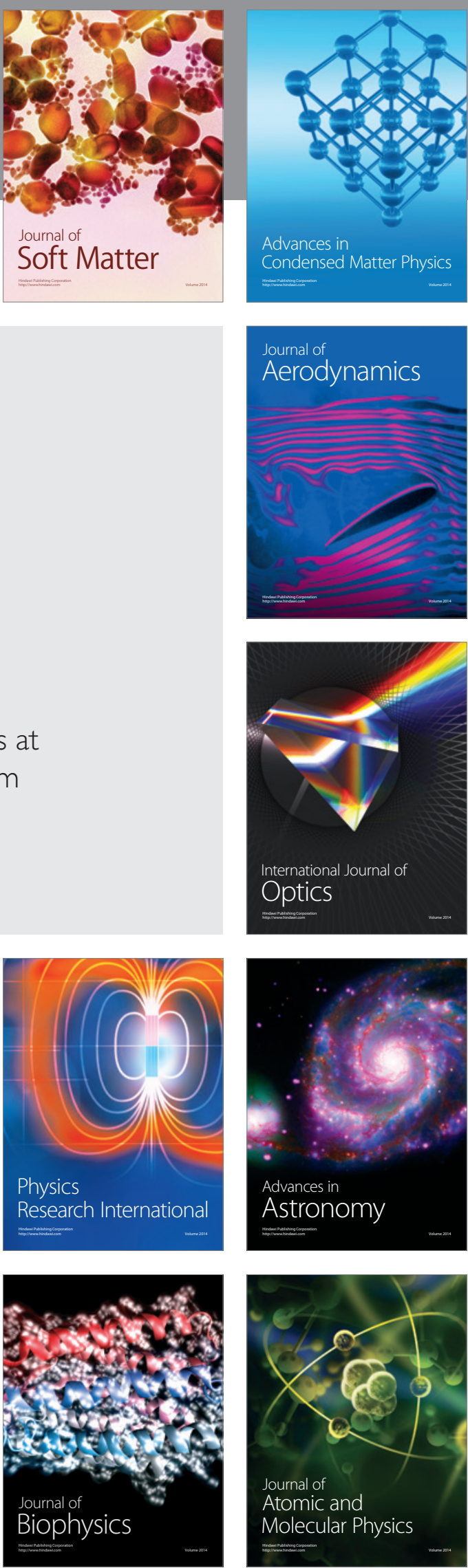